\title{
USO DA TERMOGRAFIA INFRAVERMELHA NA ANÁLISE DA TERMORREGULAÇÃO DE CAVALO EM TREINAMENTO
}

\author{
DANIELla J. DE MOURA ${ }^{1}$, ANA PAULA DE A. MAIA ${ }^{2}$, RIMENA DO A. \\ VERCELLINO $^{3}$, BRENDA B. L. MEDEIROS ${ }^{4}$, JULIANA SARUBBI ${ }^{5}$, PAULO R.GRISKA ${ }^{6}$
}

RESUMO: O estresse térmico em equinos aciona mecanismos termorregulatórios, como mudanças no fluxo sanguíneo periférico, para a manutenção da homeostase corporal. A termografia infravermelha permite detectar estas alterações, sendo uma ferramenta útil para avaliar o estresse em animais. Assim, o objetivo deste estudo foi avaliar o uso da termografia infravermelha na termorregulação de equino em condição de treinamento. Foi utilizado um cavalo anglo-árabe, exercitado uma vez ao dia. Foram captadas imagens termográficas da axila, garupa, peito e virilha do cavalo e registrados os parâmetros fisiológicos antes e após exercício e $0 ; 5$ e 10 minutos após o banho, durante 8 dias. A temperatura, a umidade relativa e a velocidade do ar foram monitoradas. A temperatura de superfície da garupa e do peito não diferiu entre os tratamentos, indicando baixa participação destas partes na termorregulação. Em contrapartida, a temperatura superficial da axila e da virilha aumentou após o exercício e diminuiu após o banho, sugerindo evidência dos mecanismos vasomotores para a troca térmica do cavalo. Comportamento semelhante foi observado para as variáveis fisiológicas, o que demonstra tentativas orgânicas do organismo para sair das condições de estresse térmico. Concluiu-se que a termografia infravermelha permitiu determinar com precisão a temperatura de superfície corporal do cavalo, sendo possível inferir sobre a termorregulação.

PALAVRAS-CHAVE: equinos, estresse térmico, parâmetros fisiológicos, temperatura de superfície.

\section{INFRARED THERMOGRAPHY TO EVALUATE THE TRAINING HORSE THERMOREGULATION}

\begin{abstract}
Heat-regulation mechanisms, such as changes in peripheral blood flow, are activated by thermal stress to maintain body homeostasis. The infrared thermography enables to identify changes in blood flow and it has been valuable for recognizing stress in animals. This research aimed to evaluate the use of infrared thermography in the training horse thermoregulation. An Anglo-Arab horse was studied and exercised once a day. Infrared thermography images of horse's armpit, croup, breast and groin and physiological parameters were taken before and after exercise and 0,5 and 10 minutes after shower for eight days. The air temperature, relative humidity and air velocity were also registered. There were no differences between the surface temperature of croup and breast and the treatments, implying low participation in thermoregulation. However, the armpit and groin temperature increased after the exercise and decreased after shower, suggesting that vasomotor mechanisms were activated to heat exchange. Similar results were found for physiological parameters which show organism thermal responses for heat loss. It was concluded that infrared thermography allowed accuracy in determining the horse body surface temperature and it was possible to infer on thermoregulation.
\end{abstract}

KEYWORDS: equine, thermal stress, physiological parameters, surface temperature.

\footnotetext{
${ }^{1}$ Professora Doutora da Faculdade de Engenharia Agrícola da UNICAMP.

${ }^{2}$ Zootecnista, Doutoranda da Faculdade de Engenharia Agrícola da UNICAMP.

${ }^{3}$ Zootecnista, Mestranda da Faculdade de Engenharia Agrícola da UNICAMP.

${ }^{4}$ Doutoranda da Faculdade de Engenharia Agrícola da UNICAMP.

${ }^{5}$ Professora Doutora, Universidade Federal de Santa Maria, Câmpus CESNORS, Palmeira das Missões - RS.

${ }^{6}$ Professor M.Sc., Faculdade de Jaguariúna.

Recebido pelo Conselho Editorial em: 4-10-2010

Aprovado pelo Conselho Editorial em: 6-12-2010
} 


\section{INTRODUÇÃO}

Uma das principais características do treinamento e provas esportivas de cavalos é a grande influência do ambiente térmico no desempenho esportivo, por serem atividades ao ar livre, permitindo, assim, a exposição direta às variáveis climáticas. E ainda, a resposta térmica do organismo ao exercício é afetada diretamente pelas condições ambientais (McCUTCHEON \& GEOR, 2008).

De acordo com a literatura científica mundial, o resultado do exercício físico é a produção de calor. Conforme CARVALHO \& MARA (2010), durante um esforço físico, em torno de $20 \%$ da energia química proveniente da oxidação dos nutrientes transforma-se em energia mecânica, responsável pelo movimento, e o restante transforma-se em energia térmica. Esta energia térmica, que se acumula durante a prática do exercício, elevando a temperatura corporal, precisa ser dissipada através dos mecanismos termorregulatórios do indivíduo.

A quantidade de calor dissipada vai ser influenciada pela condição do ambiente térmico. As vias de dissipação de calor mais eficientes nos equinos são evaporação, condução e convecção. Em temperaturas mais amenas, a principal via de dissipação do calor é a forma sensível (condução e convecção), enquanto sob estresse por calor, a perda de calor latente por evaporação é a mais eficiente (PERISSINOTTO et al., 2006).

De acordo com TITTO et al. (2009), a perda de calor por evaporação tem como vias termolíticas a sudação e o ofego. Já o método de resfriamento, realizado pelos banhos, praticado nos cavalos de esporte, baseia-se no princípio de condução. E, segundo McCONAGHY et al. (1996), nos cavalos atletas, a principal via para transferência de calor por convecção é o ajuste da circulação sanguínea, que direciona o calor do músculo para a pele e para o sistema respiratório, fazendo, desta forma, com que o sistema cardiovascular seja o principal executor da termorregulação.

RIBEIRO et al. (2008) expõem que os animais utilizam a vasodilatação periférica, ou seja, o aumento do fluxo sanguíneo para a superfície corporal, como um processo para a manutenção da homeotermia, ocasionando aumento na temperatura da superfície animal. Esta vasodilatação facilita a troca de calor do animal com o meio ambiente por processos sensíveis, e a eficácia deste depende do gradiente térmico entre o corpo do animal e a temperatura ambiente (McCUTCHEON \& GEOR, 2008).

A maioria dos estudos (PERRONE et al., 2006; PUOLI FILHO et al., 2007; OLIVEIRA et al., 2008; BRANDI et al., 2009) que são desenvolvidos para verificar o efeito do ambiente térmico e do exercício sobre a termorregulação de equinos, avalia a frequência cardíaca e respiratória, e a temperatura retal. No entanto, há poucas pesquisas que relacionam a temperatura de superfície de equinos com o ambiente térmico e o exercício.

Segundo STEWART et al. (2005), a temperatura infravermelha pode detectar alterações no fluxo sanguíneo periférico, podendo ser uma ferramenta útil para avaliar o estresse em animais. KNÍŽKOVÁ et al. (2007) citam que a câmera termográfica é capaz de detectar variações mínimas de temperatura com precisão. Com isso, a utilização da análise de termografia infravermelha torna possível identificar pontos de valores distintos de temperatura radiante e tem sido valiosa para o reconhecimento de eventos fisiológicos em animais (BOUZIDA et al., 2009).

O presente trabalho tem como objetivo avaliar o uso da termografia infravermelha na análise da termorregulação de equino em condição de treinamento.

\section{MATERIAL E MÉTODOS}

O experimento foi conduzido no município de Campinas - SP, na Hípica Rio das Pedras, entre setembro de 2009 e março de 2010, contabilizando 8 dias de coleta, realizadas em condições climáticas aproximadamente semelhantes, durante o período mais quente do dia (entre $13 \mathrm{~h} \mathrm{e} 15 \mathrm{~h}$ ), com a temperatura ambiente média em torno de $31,9 \pm 2,9^{\circ} \mathrm{C}$, com desvio-padrão de 3,76 , a 
umidade relativa em 43,5 $\pm 11,0 \%$, com desvio-padrão de 13,7 , e a velocidade do ar em torno de $0,52 \pm 0,46 \mathrm{~m} \mathrm{~s}^{-1}$, com desvio-padrão de 0,52 .

Utilizou-se como unidade experimental um cavalo anglo-árabe, com 8 anos de idade, pelagem castanho-escura e massa corporal de $\pm 420 \mathrm{~kg}$. Esse cavalo foi exercitado no estilo "ring-work" (exercício em pista), durante 30 minutos, em cada dia de coleta.

Os tratamentos consistiram em cinco períodos distintos: antes do exercício (AE), após o exercício (APE), após o banho 0 minuto (AB0), após o banho 5 minutos (AB5) e após o banho 10 minutos (AB10), de acordo com o protocolo de banho das provas de enduro. O banho foi realizado em local próprio da hípica, com o uso de uma mangueira, molhando todo o corpo do animal. A temperatura média da água foi de $19,2 \pm 1,6^{\circ} \mathrm{C}$.

\section{Variáveis ambientais}

Em todos os tratamentos, o ambiente térmico foi monitorado, coletando-se a temperatura do ar (Tbs, ${ }^{\circ} \mathrm{C}$ ), a umidade relativa (UR, \%) e a velocidade do ar (Var, $\mathrm{m} \mathrm{s}^{-1}$ ). Os dados de UR foram coletados por meio do equipamento THDL 400 (Instrument $^{\circledR}$ ), com amplitude de 25 a $95 \%$ e precisão de $\pm 5 \%$, conforme mostra a Figura 1. Para a coleta da Tbs e Var, foi utilizado um anemômetro de fio quente VelociCalc $\left(\mathrm{TSI}_{\mathrm{TM}}{ }^{\circledR}\right)$, com escala de leitura para temperatura de -18 a $93{ }^{\circ} \mathrm{C}$ e resolução $0,1^{\circ} \mathrm{C}$, e velocidade do ar com amplitude de 0 a $30 \mathrm{~m} \mathrm{~s}^{-1}$, resolução de velocidade de $0,01 \mathrm{~m} \mathrm{~s}^{-1}$ e exatidão de $\pm 0,015 \mathrm{~m} \mathrm{~s}^{-1}$, ilustrados na Figura 2.

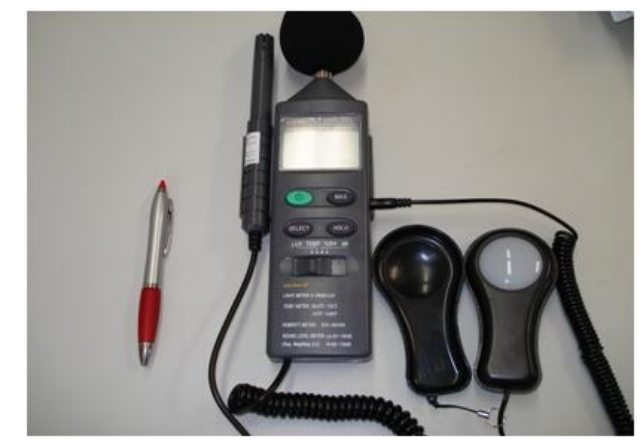

FIGURA 1. Equipamento THDL 400, da marca Instrutherm $^{\circledR}$. Equipment THDL 400, Instrutherm ${ }^{\circledR}$ brand.

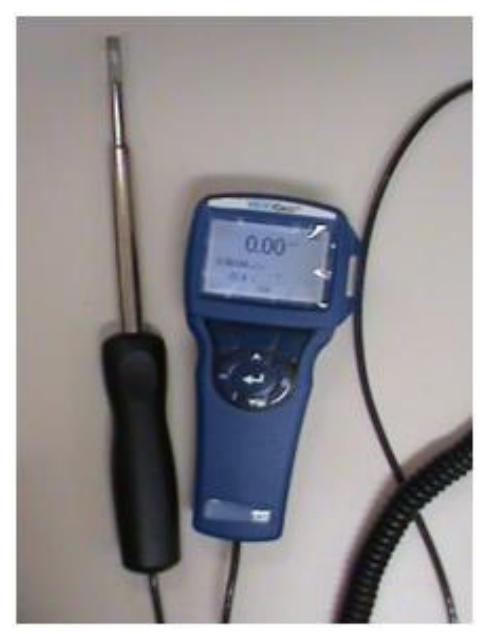

FIGURA 2. Anemômetro de fio quente VelociCalc ${ }^{\circledR}$ da marca $\operatorname{TSI}_{\mathrm{TM}}{ }^{\circledR}$. VelociCalc $\AA$ hot-wire anemometer, TSI $_{\mathrm{TM}}{ }^{\circledR}$ brand. 
Com o intuito de caracterizar se o ambiente térmico durante o período de coleta foi estressante ou não para o cavalo, utilizou-se o Índice de Conforto (IC), empregado por Jones (2009), determinado pela seguinte fórmula:

$$
\mathrm{IC}=\text { Temperatura do ar }\left({ }^{\circ} \mathrm{F}\right)+\text { Umidade Relativa }(\%)
$$

Segundo este autor, se o IC for menor que 130, o sistema termorregulatório do animal não será acionado. Acima disso, dependendo do valor, a dissipação de calor poderá ser prejudicada.

As variáveis respostas foram: frequência cardíaca (FC), frequência respiratória (FR), temperatura retal (TR) e temperatura de superfície (TS) da axila, garupa, peito e virilha do cavalo.

\section{Parâmetros fisiológicos}

A FC (bat $\min ^{-1}$ ) foi obtida pela contagem dos batimentos cardíacos com a utilização de estetoscópio manual, e a FR ( $\mathrm{mov} \mathrm{min}^{-1}$ ) foi medida pela contagem dos movimentos do flanco do animal, ambas mensuradas durante um minuto. Para dados de TR $\left({ }^{\circ} \mathrm{C}\right)$, um termômetro de mercúrio foi mantido no reto do cavalo durante aproximadamente dois minutos.

\section{Temperatura de superfície}

Utilizou-se uma câmera termográfica Thermal Imager (Testo $880^{\circledR}$ ), ilustrada na Figura 3, com precisão de $\pm 0,1^{\circ} \mathrm{C}$ e intervalo de espectro infravermelho de 7,5 a $13 \mu \mathrm{m}$, para a coleta da TS das diferentes partes do corpo do cavalo.

As imagens foram analisadas no próprio software da câmera (Testo IRSoft Software), com a escala definida na opção de paleta frio / quente e com a temperatura variando entre 17 e $40{ }^{\circ} \mathrm{C}$. A TS foi calculada considerando uma área predeterminada para as seguintes partes do corpo do cavalo: axila (Figura 4), garupa (Figura 5), peito (Figura 6) e virilha (Figura 7).

A região da axila e da virilha foi escolhida por ser altamente vascularizada (McCUTCHEON \& GEOR, 2008) e com poucos estudos a respeito. A garupa é uma região muito exposta às variáveis ambientais e foi usada como referência no estudo de KOHN et al. (1999). Já a região do peito, por apresentar alta variabilidade térmica e ser estimada como representativa da média da superfície corporal (MARLIN et al., 1998).

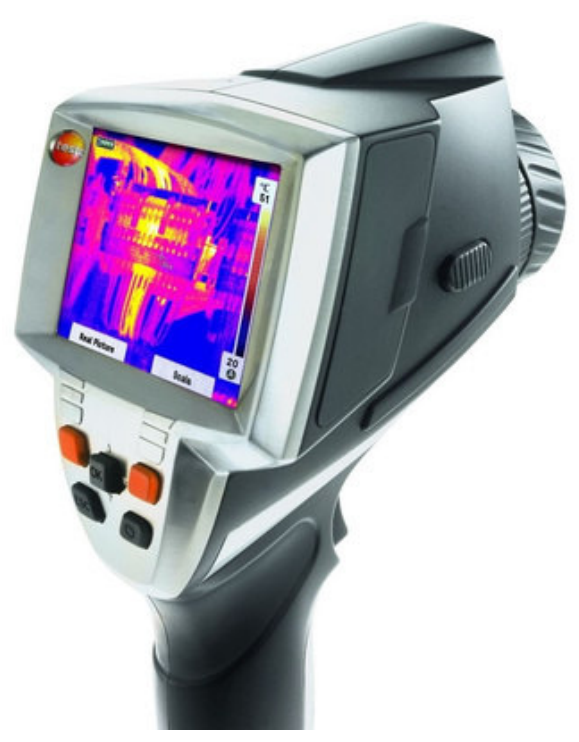

FIGURA 3. Câmera de infravermelho - Testo 880. Infrared Camera - Testo 880.

Em todas as coletas, a câmera termográfica foi posicionada a uma distância padronizada, para melhor focar e fotografar todas as partes coletadas, sendo $0,70 \mathrm{~m}$ da axila e da virilha e $1,60 \mathrm{~m}$ do peito e da garupa. 
O índice de emissividade (ع) utilizado para este experimento foi de 0,95 para todas as regiões analisadas,o mesmo valor usado por AUTIO et al. (2006) em experimento similar.
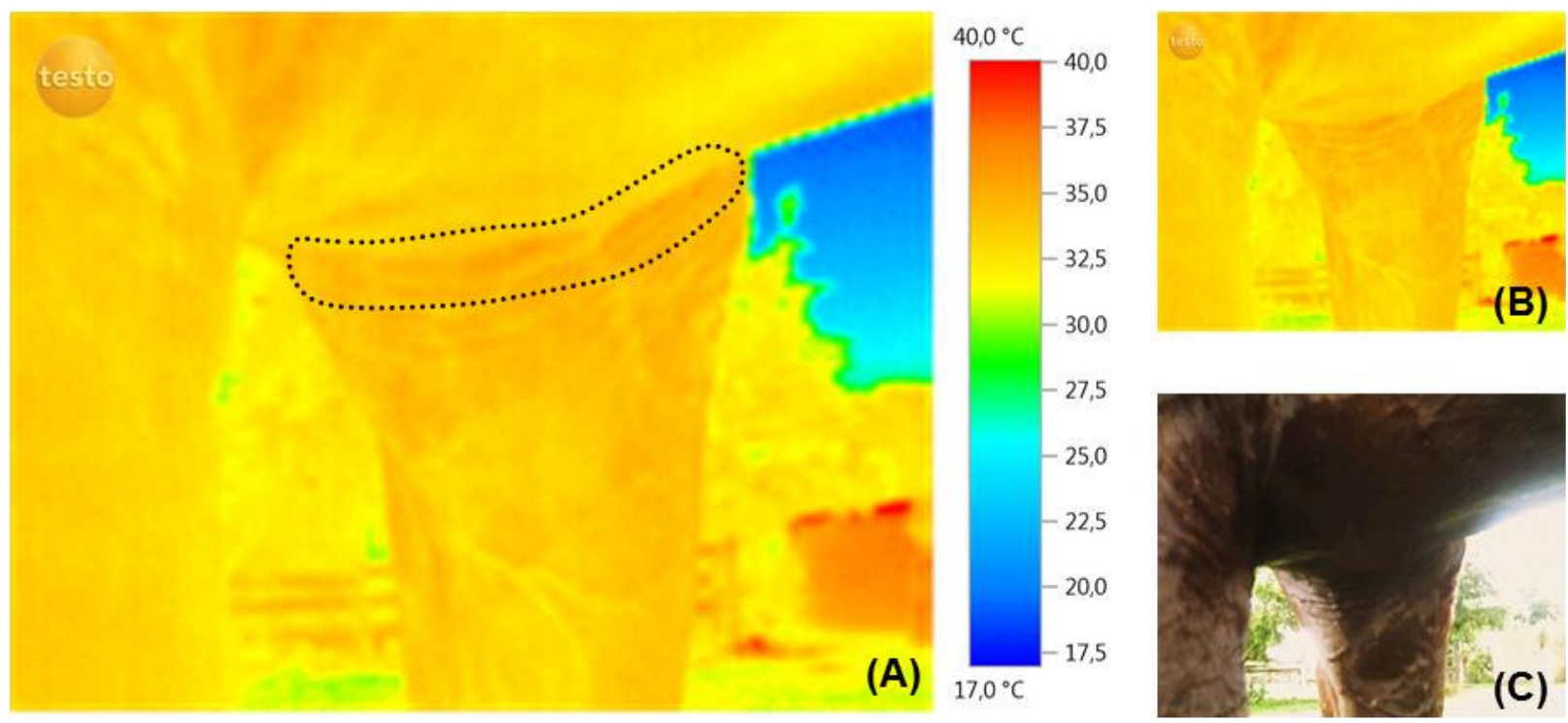

FIGURA 4. Área correspondente à região onde a temperatura superficial foi extraída (A); imagem termográfica da axila (B), e imagem real da axila (C). Area corresponding to the region where the surface temperature was obtained (A), thermographic image of the armpit (B), and real image of the armpit (C).
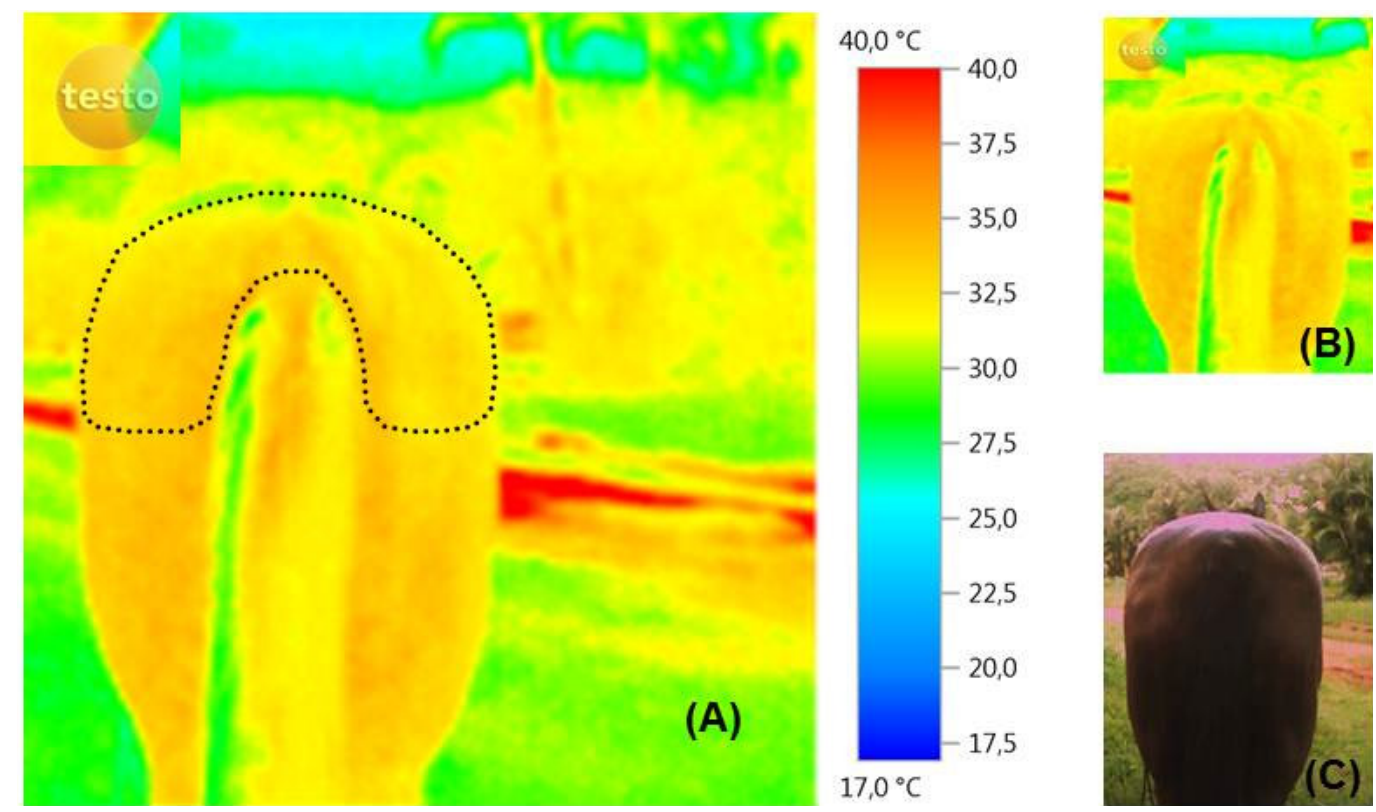

FIGURA 5. Área correspondente à região onde a temperatura superficial foi extraída (A); imagem termográfica da garupa (B), e imagem real da garupa (C). Area corresponding to the region where the surface temperature was obtained $(\mathrm{A})$, thermographic image of the croup (B), and real image of the croup (C). 

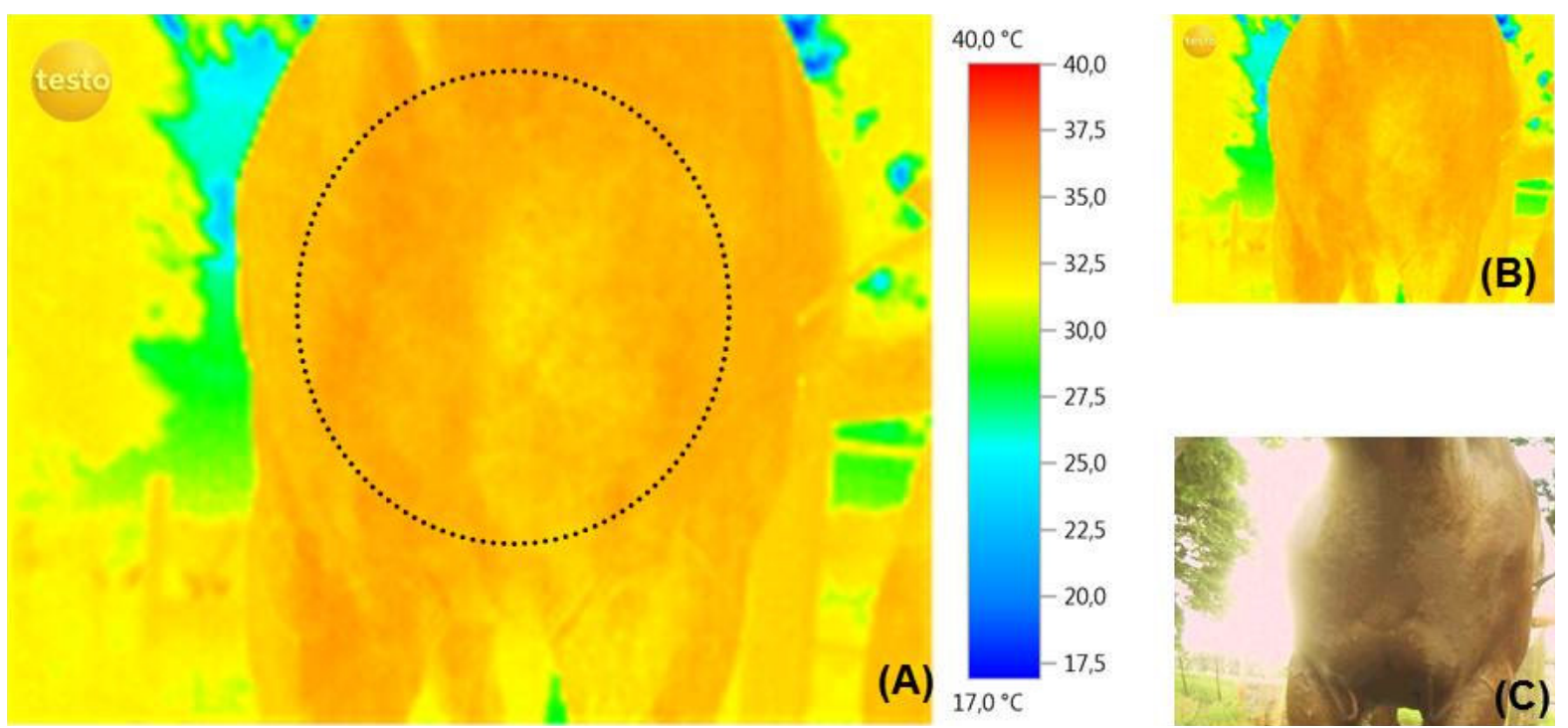

FIGURA 6. Área correspondente à região onde a temperatura superficial foi extraída (A); imagem termográfica do peito (B), e imagem real do peito (C). Area corresponding to the region where the surface temperature was obtained $(A)$, thermographic image of the breast $(B)$, and real image of the breast $(C)$.
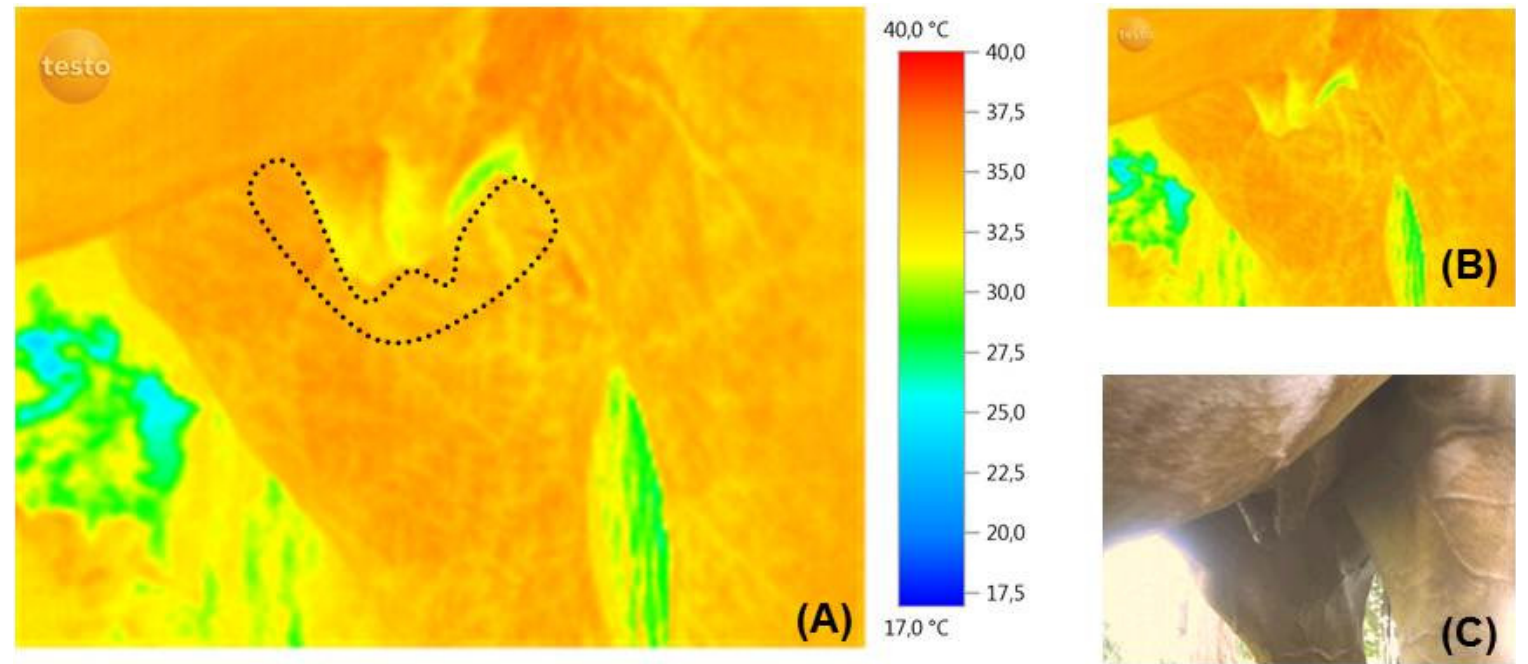

FIGURA 7. Área correspondente à região onde a temperatura superficial foi extraída (A); imagem termográfica da virilha (B), e imagem real da virilha (C). Area corresponding to the region where the surface temperature was obtained $(A)$, thermographic image of the groin $(B)$, and real image of the groin $(C)$.

\section{Análise estatística}

Para a análise estatística do comportamento das TS e dos parâmetros fisiológicos, recorreu-se à análise de variância (ANOVA) e à comparação de médias, pelo teste de Tukey, a 5\% de significância. O IC e a Var foram investigados como covariáveis, mas não foram significativos, sendo retirados do modelo. Para o tratamento destes dados, o Sistema para Análises Estatísticas e Genéticas - SAEG (UNIVERSIDADE FEDERAL DE VIÇOSA, 2007) foi utilizado.

\section{RESULTADOS E DISCUSSÃO}

Durante o período experimental, o IC calculado foi de 133,1 $\pm 8,0$, com desvio-padrão de 10,4, caracterizando o ambiente como de desconforto térmico por calor, segundo JONES (2009). 
Os tratamentos não influenciaram $(\mathrm{P}>0,05)$ a TS da garupa e do peito (Tabela 1$)$. Tendo em vista que o exercício aumenta a produção de calor corporal e que a termorregulação através de ajustes do fluxo sanguíneo é eficiente para manter a estabilidade térmica (McCUTCHEON \& GEOR, 2008), estes resultados podem sugerir baixa capacidade vasomotora destas partes do corpo, apesar do aumento do fluxo sanguíneo em função do estresse e do exercício, e da rica vascularização e representação destas regiões. Em contrapartida, McCONAGHY et al. (1996), ao mensurarem a distribuição sanguínea de pôneis submetidos ao estresse por calor, verificaram o aumento de $300 \%$ na circulação de sangue no peito e na garupa, demonstrando capacidade destas regiões em aumentar o fluxo sanguíneo, atendendo às necessidades termorregulatórias.

TABELA 1. Médias e coeficiente de variação (CV) das temperaturas de superfície e frequências cardíaca (FC) e respiratória (FR) e temperatura retal (TR) de equino em função dos tratamentos. Mean values and variation coefficient $(\mathrm{CV})$ of the surface temperatures, heart rate $(\mathrm{FC})$, respiratory rate $(\mathrm{FR})$, and rectal temperature (TR) of equine in treatments.

\begin{tabular}{lccccccc}
\hline \multirow{2}{*}{ Variável } & AE & APE & AB0 & AB5 & AB10 & CV $(\%)$ & Desvio-Padrão \\
\cline { 2 - 8 } & & \multicolumn{7}{c}{ TS partes do corpo $\left({ }^{\circ} \mathrm{C}\right)$} \\
Axila & $34,8 \mathrm{~b}$ & $35,2 \mathrm{a}$ & $33,8 \mathrm{bc}$ & $33,7 \mathrm{bc}$ & $32,9 \mathrm{c}$ & 3,8 & 1,7 \\
Garupa & $33,5 \mathrm{a}$ & $35,0 \mathrm{a}$ & $32,5 \mathrm{a}$ & $32,5 \mathrm{a}$ & $33,9 \mathrm{a}$ & 8,0 & 2,9 \\
Peito & $33,7 \mathrm{a}$ & $34,8 \mathrm{a}$ & $33,1 \mathrm{a}$ & $33,4 \mathrm{a}$ & $33,2 \mathrm{a}$ & 5,0 & 2,0 \\
Virilha & $34,2 \mathrm{~b}$ & $35,3 \mathrm{a}$ & $33,2 \mathrm{~b}$ & $33,9 \mathrm{~b}$ & $33,8 \mathrm{~b}$ & 4,0 & 1,9 \\
\hline & \multicolumn{7}{c}{ Variáveis fisiológicas } \\
FC (bpm) & $35,6 \mathrm{~b}$ & $57,8 \mathrm{a}$ & $45,6 \mathrm{~b}$ & $43,1 \mathrm{~b}$ & $39,3 \mathrm{~b}$ & 16 & 10,2 \\
FR (mpm) & $17,3 \mathrm{~b}$ & $30,2 \mathrm{a}$ & $20,6 \mathrm{ab}$ & $18,9 \mathrm{ab}$ & $16,7 \mathrm{~b}$ & 43 & 9,8 \\
TR $\left({ }^{\circ} \mathrm{C}\right)$ & $37,3 \mathrm{c}$ & $38,9 \mathrm{a}$ & $38,5 \mathrm{ab}$ & $38,2 \mathrm{~b}$ & $37,9 \mathrm{~b}$ & 0,9 & 0,6 \\
\hline As
\end{tabular}

As médias seguidas por letra diferentes na mesma linha diferem entre si $(\mathrm{P}>0,05)$, pelo teste de Tukey.

O comportamento da TS da garupa e do peito observado neste estudo indica que a participação destas regiões na termorregulação pode apresentar uma relação mais estreita com a transferência de calor por condução da massa muscular do que com os eventos vasomotores, já que, neste trabalho, não foi medida a temperatura intramuscular e sim a de superfície. Esta proposição pode ser sustentada, tendo em vista que a região da garupa é onde se concentram os tecidos subjacentes que representam a maior e mais ativa massa muscular do organismo no exercício, assim como a massa muscular da região do peito, aumentando sua atividade em função de alterações fisiológicas, como o aumento da FC e FR (ETCHICHURY, 2008). E ainda, a região peitoral pode ser resfriada pelo ofego, não necessitando ser uma via termorregulatória aparente.

As TSs da axila e da virilha aumentaram estatisticamente $(\mathrm{P}<0,05)$ APE e diminuíram $\mathrm{AB}$, voltando ao valor inicial, não diferindo em função do repouso. Considerando estes resultados, o aumento das TSs pode ser atribuído à capacidade de aumento sanguíneo, ou seja, vasodilatação periférica destas regiões (RIBEIRO et al., 2008), por serem altamente vascularizadas, onde os mecanismos vasomotores deveriam ser mais evidentes (CUNNIGHAM, 2002). E ainda, a redução das TSs em virtude do banho pode ser atribuída a uma vasoconstrição periférica, associada a uma transferência de calor da pele do cavalo para a água por condução (ETCHICHURY, 2008), contribuindo para uma troca térmica mais eficiente. Deste modo, pode-se inferir que estas regiões anatômicas participam ativamente da troca de calor do cavalo.

Com relação às variáveis fisiológicas, é possível observar, na Tabela 1 , que a FC aumentou $(\mathrm{P}<0,05)$ APE, indo além dos níveis basais de 32 a 44 bpm de acordo com CUNNINGHAM (2002). Ainda, pode-se verificar que a FC reduziu $(\mathrm{P}<0,05)$ imediatamente após o banho, voltando estatisticamente ao valor inicial. Com estes resultados, é possível aferir que a elevação dos bpm 
pode ser explicada pelo aumento do fluxo sanguíneo, consequente da vasodilatação periférica, requerida para dissipação de calor e que é comprovada pelo aumento das TSs da axila e da virilha.

Comportamento semelhante aos dados da FC foi observado para FR; no entanto, para todos os tratamentos, a FR ficou ligeiramente acima da preconizada na literatura, de 8 a 16 respirações por minuto (CUNNINGHAM, 2002).

Segundo PIRRONE et al. (2007), os cavalos usam o sistema respiratório para perder calor por resfriamento evaporativo, e, para aumentar esta perda, eles hiperventilam por aumento da FR. SILVA et al. (2005) e TITTO et al. (2009) ainda relatam que a FR é o segundo mecanismo mais importante de perda de calor pelos equinos quando aumenta o estresse térmico, seja pelo ambiente, seja pelo exercício. Assim, as FRs encontradas neste estudo acima da basal podem ser devido ao ambiente térmico já estar estressante para o animal (IC > 130), acionando este importante mecanismo termorregulatório e, ainda, que o exercício acentuou o estresse sofrido pelo cavalo, por aumentar a produção de calor, já que a FR se elevou após o exercício, sendo um valor superior ao do repouso.

De acordo com o resultado da análise estatística das variáveis fisiológicas (Tabela 1), pode-se observar que a FR apresentou o maior coeficiente de variação (43\%). Resultados semelhantes foram encontrados por PALUDO et al. (2002), PUOLI FILHO et al. (2007) e OLIVEIRA et al. (2008). OLIVEIRA et al. (2008) explicam que a FR é o mecanismo termorregulatório mais facilmente usado quando o animal está submetido ao estresse térmico, denotando, assim, ser passível de maior variação.

Segundo TITTO et al. (2009), alterações nas FC e FR podem evidenciar tentativas orgânicas para sair das condições de estresse térmico a que o cavalo está submetido.

Tanto para FC quanto para FR, o banho foi eficiente na recuperação do animal após exercício. O comportamento da FC e FR analisada neste estudo corrobora a proposição de McCUTCHEON \& GEOR (2008) de que os seus ajustes em equinos são muito rápidos e que estes sistemas participam ativamente da termorregulação destes animais, embora eles também aumentem a produção de calor.

Quanto à TR, é possível verificar, também, um aumento em APE, evidenciando que o exercício e a elevação da FC e da FR contribuem para o aumento da produção de calor corporal, influenciando a TR. Além disso, este aumento pode sugerir que o esforço do organismo para dissipar calor, através da vasodilatação, da FC e da FR, não foi suficiente a ponto de impedir uma variação da TR. Resultados semelhantes foram observados por TITTO et al. (2009).

Com relação ao resfriamento, pode-se ressaltar que TR reduziu em $\mathrm{AB} 5$ e $\mathrm{AB} 10$, mas não foi capaz de retornar ao valor inicial $\left(37,4^{\circ} \mathrm{C}\right)$, embora os valores encontrados após banho tenham permanecido na faixa basal permitida de 37,2 a $38,2^{\circ} \mathrm{C}$ (CUNNIGHAM, 2002). Estes dados são justificados pela conjectura de CUNNIGHAM (2002) de que, quando os mecanismos termorregulatórios são intensificados, a temperatura corporal pode estabilizar-se, porém em nível mais elevado.

Analisando os dados de variáveis fisiológicas, é possível sugerir que o banho após o exercício mostrou-se hábil na recuperação do cavalo, embora o resfriamento obtido desta maneira, no qual a transferência de calor da pele do cavalo para água é por condução, não seja a via termolítica mais eficiente (MORGAN et al.,1997). Esta proposição pode ser justificada pelo relato de SILVA et al. (2005) e PERRONE et al. (2006), que afirmam que a capacidade do animal em se recuperar após o término do exercício pode ser determinada pelo acompanhamento da FR, TR e, principalmente, da FC, e mais rápida é a recuperação, quanto mais breve estas variáveis voltarem aos níveis basais, indicando boa condição física do animal.

Alterações da FC, FR e TR, em virtude do exercício e/ou do estresse por calor, também foram verificadas por PALUDO et al. (2002), PUOLI FILHO et al. (2007), OLIVEIRA et al.(2008) e BRANDI et al. (2009). 


\section{CONCLUSÕES}

O uso da termografia infravermelha permitiu precisão na determinação da temperatura de superfície das partes do corpo do cavalo e sua associação com a termorregulação. O comportamento das temperaturas superficiais da axila e da virilha mostrou capacidade de aumento e diminuição da vascularização periférica destas regiões, reafirmando sua importância na homeotermia dos equinos. O banho após o exercício mostrou-se um método eficiente para auxiliar a troca térmica do cavalo com o ambiente.

\section{REFERÊNCIAS}

AUTIO, E.; NESTE, R.; AIRAKSINEN, S.; HEISKANEN, M. Measuring the heat loss in horses in different seasons by infrared thermography. Journal of Applied Animal Welfare Science, Mahwah, v.9, n.3, p.211-221, 2006.

BOUZIDA, N.; BENDADA, A.; MALDAGUE, X.P. Visualization of body thermoregulation by infrared imaging. Journal of Thermal Biology, Oxford, v.34, n.3, p.120-126, 2009.

BRANDI, R.A.; FURTADO, C.E.; MARTINS, E.N.; FREITAS, E.V.V.; LACERDA NETO, J.C.; QUEIROZ NETO, A.; RIBEIRO, L.B. Desempenho de equinos submetidos a enduro alimentados com níveis de óleo de soja na dieta. Revista Brasileira de Saúde e Produção Animal, Salvador, v.10, n.2, p.311-321, 2009.

CARVALHO, T.; MARA, L.S. Hidratação e nutrição no esporte. Revista Brasileira de Medicina do Esporte, Rio de Janeiro, v.16, n.2, p. 33-40. 2010.

CUNNINGHAM, J.G. Termorregulação. In: Tratado de fisiologia veterinária. 3.ed. São Paulo: Guanabara Koogan, 2002. p.550-561.

ETCHICHURY, M. Termorregulação em cavalos submetidos a diferentes métodos de resfriamento pós-exercício. 2008. 103 f. Tese (Doutorado em Zootecnia) - Universidade de São Paulo, Faculdade de Zootecnia e Engenharia de Alimentos, Pirassununga, 2008.

JONES, S. Horsback riding in the dog days. Animal Science e-News, v.2, n.3, p. 3-4,2009.

Disponível em: <http://www.aragriculture.org/News/animal_science_enews/2009/pdf/july2009. pdf $>$. Acesso em: 5 maio 2010.

KNÍŽKOVÁ, I.; KUNC, P.; GÜRDÍL, G.A.K.; PINAR, Y.; SELVÍ, K.Ç. Applications of infrared thermography in animal production. Journal of the Faculty of Agriculture, Kyushu, v.22, n.3, p.329-336, 2007.

KOHN, C.W.; HINCHCLIFF, K.W.; MCKEEVER, K.H. Evaluation of washing with cold water to facilitate heat dissipation in horses exercised in hot, humid conditions. American Journal of Veterinary Research, Schaumburg, v.60, n.3, p.299-305, 1999.

OLIVEIRA, L.A.; CAMPEL, J.E.G.; AZEVEDO, D.M.M.R.; COSTA, A.P.R.; TURCO, S.H.N.; MOURA, J.W.S. Estudo de respostas fisiológicas de equinos sem raça definida e da raça quarto de milha às condições climáticas de Teresina, Piauí. Ciência Animal Brasileira, Goiânia, v.9, n.4, p.827-838, 2008.

MARLIN, D.J.; SCOTT, C.M.; ROBERTS, C.A.; CASAS, I.; HOLAH, G.; SCHROTER, R. Post exercise changes in compartmental body temperature accompanying intermittent cold water cooling in the hyperthermic horse. Equine Veterinary Journal, Borough Green, n.30, p.28-34, 1998.

McCONAGHY, F.F.; HODGSON, D.R.; ROSE, R.J.; HALES, J.R.S. Redistribution of cardiac output in response to heat exposure in the pony. Equine Veterinary Journal Supplement, Newmarket, v.22, p.42-46, july, 1996. 
McCUTCHEON, L.J.; GEOR, R.J. Thermoregulation and exercise-associated heat stress. In: HINCHCLIFF, K.W.; GEOR, R.J.; KANEPS, A.J. Equine exercise physiology: the science of exercise in the athletic horse. Philadelphia: Elsevier, 2008, p.382-386.

MORGAN, K.; EHRLEMARK, A.; SÄLLVIK, K. Dissipation of heat from standing horses exposed to ambient temperatures between $-3{ }^{\circ} \mathrm{C}$ and $37^{\circ} \mathrm{C}$. Journal of Thermal Biology, Great Britain, v.22, n.3, p.177-186, 1997.

PALUDO, G.R.; McMANUS, C.; MELO, R.Q.; CARDOSO, A.G.; MELLO, F.P.S.; MOREIRA, M.; FUCK, B.H. Efeito do estresse térmico e do exercício sobre os parâmetros fisiológicos de cavalos do exército brasileiro. Revista Brasileira de Zootecnia, Viçosa-MG, v.31, n.3, p.1.130$1.142,2002$.

PERISSONOTO, M.; MOURA, D.J.; MATARAZZO, S.V.; SILVA, I.J.O.; LIMA, K.A.O. Efeito da utilização de sistemas de climatização nos parâmetros fisiológicos do gado leiteiro. Engenharia Agrícola, Jaboticabal, v.26, n.3, p.663-671, 2006.

PERRONE, G.M.; CAVIGLIA, J.F.; PÉREZ, A.; FIDANZA, M.; MARQUEZ, A.; CATELLI, J.L.; GONZÁLEZ, G. Cambios em las variables fisiológicas en equinos compitiendo en una prueba combinada. Anales de Veterinaria, Murcia, v.22, p.35-42, 2006.

PIRRONE, F.; ALBERTINI, M.; CLEMENT, M.G.; LAFORTUNA, C.L. Respiratory mechanics in Standardbred horses with sub-clinical inflammatory airway disease and poor athletic performance. The Veterinary Journal, London, v.173, p.144-150, 2007.

PUOLI FILHO, J.N.P.; BARROS NETO, T.L.; RODRIGUES, P.H.M.; GARCIA, H.P.L.

Parâmetros fisiológicos do desempenho de cavalos de alta performance hidratados voluntariamente com água ou solução isotônica contendo carboidrato. Brazilian Journal of Veterinary Research and Animal Science, São Paulo, v.44, n.2, p.122-131, 2007.

RIBEIRO, N.L.; FURTADO, D.A.; MEDEIROS, A.N.; RIBEIRO, M.N.; SILVA, R.C.B.; SOUZA, C.M.S. Avaliação dos índices de conforto térmico, parâmetros fisiológicos e gradiente térmico de ovinos nativos. Revista de Engenharia Agrícola, Jaboticabal, v.28, n.4, p.614-623, 2008.

SILVA, L.A.C.; SANTOS, S.A.; SILVA, R.A.S.; McMANUS, C.; PETZOLD, H. Adaptação do cavalo pantaneiro ao estresse da lida diária de gado no Pantanal, Brasil. Archivos de Zootecnia, Córdoba, v.54, p.509-513, 2005.

STEWART, M.; WEBSTER, J.R.; SCHAEFER, A.L.; COOK, N.J.; SCOTT, S.L. Infrared thermography as a non-invasive tool to study animal welfare. Animal Welfare, South Mimms, v.14, p.319-325, 2005.

TITTO, E.A.L.; PEREIRA, A.M.F.; TOLEDO, L.R.A.; PASSINI, R.; NOGUEIRA FILHO, J.C.M.; GOBESSO, A.A.O.; ETCHICHURY, M.; TITTO, C.G. Concentração de eletrólitos em equinos submetidos a diferentes temperaturas. Revista Brasileira de Saúde e Produção Animal, Salvador, v.10, n.1, p.236-244, 2009.

UNIVERSIDADE FEDERAL DE VIÇOSA. SAEG - Sistemas para análises estatísticas e genéticas. Versão 9.1. Viçosa-MG, 2007. 150 p. (Manual do usuário). 